\title{
Simple Self-Assembled Targeting DNA Nano Sea Urchin as a Multivalent Drug Carrier
}

\author{
Jingjing Zhang, ${ }^{\dagger}$ Zhenmeng Wang, ${ }^{\dagger}$ Yansha Gao, and Zai-Sheng $\mathrm{Wu}^{*}$ \\ Cancer Metastasis Alert and Prevention Center, Fujian Provincial Key Laboratory of Cancer \\ Metastasis Chemoprevention and Chemotherapy, National and Local Joint Biomedical Engineering \\ Research Center on Photodynamic Technologies, State Key Laboratory of Photocatalysis on Energy \\ and Environment, College of Chemistry, Fuzhou University, Fuzhou, 305108, China. \\ $\dagger$ J.Z. and Z.W. contributed equally to this work.
}

*Corresponding Author: Zai-Sheng Wu; Email, wuzaisheng@163.com (Z.S. Wu) 
Table S1. DNA oligonucleotides designed in this work.

\begin{tabular}{|c|c|c|}
\hline Note & \multicolumn{2}{|l|}{ Sequence (5'-3') } \\
\hline $\begin{array}{l}\text { Palindrome sequence } 1 \\
\text { (PS-1) }\end{array}$ & \multicolumn{2}{|l|}{ TAGGAGCTCCTA } \\
\hline PS-2 & \multicolumn{2}{|l|}{ TAGGAGCTCCTA GATGTTAACATC } \\
\hline PS-3 & \multicolumn{2}{|c|}{ TAGGAGCTCCTA GATGTTAACATC GTAAGCGCTTAC } \\
\hline PS-4 & \multicolumn{2}{|c|}{ TAGGAGCTCCTA GATGTTAACATC GTAAGCGCTTAC } \\
\hline & \multicolumn{2}{|c|}{ TCATCCGGATGA } \\
\hline Biotinylated-PS-4 (BPS-4) & \multicolumn{2}{|c|}{ TAGGAGCTCCTA GATGTTAACATC GTAAGCGCTTAC } \\
\hline & \multicolumn{2}{|c|}{ TCATCCGGATGA-Biotin } \\
\hline \multirow[t]{2}{*}{ FAM-BPS-4 } & FAM-TAGGAGCTCCTA GATGTTAACATC & GTAAGCGCTTAC \\
\hline & \multicolumn{2}{|l|}{ TCATCCGGATGA-Biotin } \\
\hline \multirow{2}{*}{$\begin{array}{l}\text { B-T-sgc8 (Biotinylated } T_{10-} \\
\text { spacer-Sgc8) }\end{array}$} & Biotin- $(\mathrm{T})_{10} \mathrm{~A}$ & GCCGCCGGGAAA \\
\hline & \multicolumn{2}{|l|}{ ATACTGTACGGT TAGA } \\
\hline \multirow[t]{2}{*}{ B-T-sgc8-FAM } & Biotin- $(T)_{10} \mathrm{~A} \quad$ TCTAACTGCTGC & GCCGCCGGGAAA \\
\hline & \multicolumn{2}{|l|}{ ATACTGTACGGT TAGA-FAM } \\
\hline \multirow[t]{2}{*}{ B-T-sgc8-Cy3 } & Biotin- $(T)_{10} \mathrm{~A} \quad$ TCTAACTGCTGC & GCCGCCGGGAAA \\
\hline & \multicolumn{2}{|l|}{ ATACTGTACGGT TAGA-Cy3 } \\
\hline \multirow[t]{2}{*}{ T-sge8 ( T $_{10}$-Spacer-Sgc8) } & $(\mathrm{T})_{10} \mathrm{~A} \quad$ TCTAACTGCTGC & GCCGCCGGGAAA \\
\hline & \multicolumn{2}{|l|}{ ATACTGTACGGT TAGA } \\
\hline \multirow[t]{2}{*}{ T-sgc8-FAM } & $(\mathrm{T})_{10 \mathrm{~A}}$ TCTAACTGCTGC & GCCGCCGGGAAA \\
\hline & \multicolumn{2}{|l|}{ ATACTGTACGGT TAGA-FAM } \\
\hline \multirow[t]{2}{*}{ T-sgc8-Cy3 } & $\begin{array}{ll}(\mathrm{T}){ }_{10} \mathrm{~A} & \text { TCTAACTGCTGC }\end{array}$ & GCCGCCGGGAAA \\
\hline & \multicolumn{2}{|l|}{ ATACTGTACGGT TAGA-Cy3 } \\
\hline \multirow{2}{*}{$\begin{array}{l}\text { T-Lib (with the scrambled } \\
\text { sequence) }\end{array}$} & $(\mathrm{T})_{10}$ CGAGTATGTTGT & CAACTGCCTTCC \\
\hline & \multicolumn{2}{|l|}{ ACTCTGATATAA ACCA } \\
\hline \multirow[t]{2}{*}{ T-Lib-FAM } & $(\mathrm{T})_{10}$ CGAGTATGTTGT & CAACTGCCTTCC \\
\hline & \multicolumn{2}{|l|}{ ACTCTGATATAA ACCA-FAM } \\
\hline \multirow[t]{2}{*}{ B-T-Lib } & Biotin- $(T)_{10} G \quad$ CGAGTATGTTGT & CAACTGCCTTCC \\
\hline & \multicolumn{2}{|l|}{ АСTCTGATATAA ACCA } \\
\hline \multirow[t]{2}{*}{ B- T-Lib-FAM } & Biotin- $(T)_{10} G$ & CAACTGCCTTCC \\
\hline & \multicolumn{2}{|l|}{ ACTCTGATATAA ACCA-FAM } \\
\hline \multirow[t]{2}{*}{ B- T-Lib-Cy3 } & Biotin- $(T)_{10} G$ & CAACTGCCTTCC \\
\hline & \multicolumn{2}{|l|}{ АСTCTGATATAA ACCA-Cy3 } \\
\hline
\end{tabular}




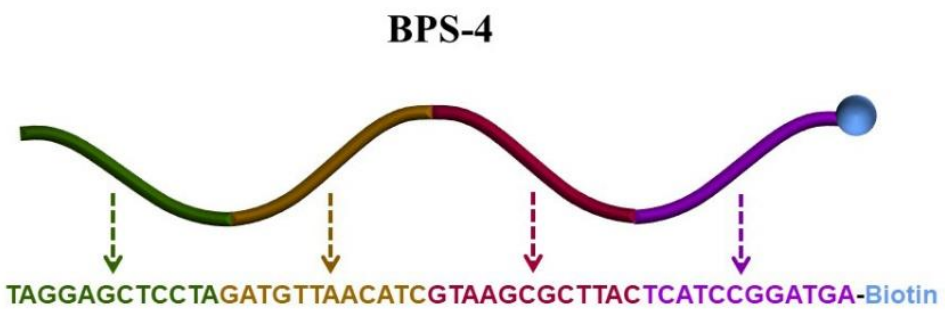

Figure S1. The schematic diagram and sequences of BPS-4. Different colors represent different palindrome domains in BPS-4. 


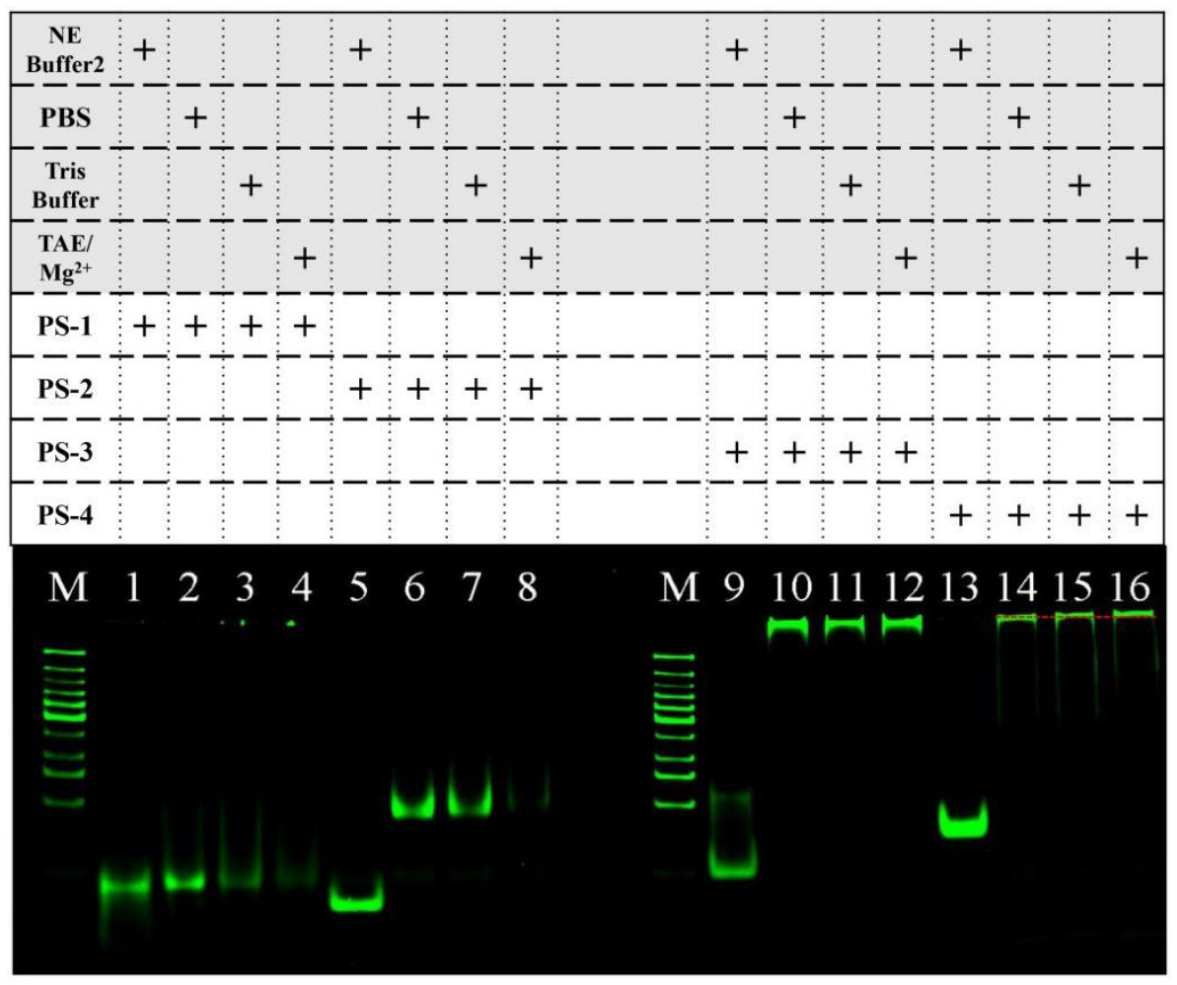

Figure S2. Optimization of the assembly buffer and the number of palindrome fragments. Native polyacrylamide gel (10\%) electrophoresis (n-PAGE) analysis of DNA nano sea urchin (NSU) assembled from different components. "+" represents that the corresponding buffer or DNA sequence component is employed for the self-assembly of NSU.

\section{Experimental procedure:}

The palindrome sequences with the different number of palindromic domains were separately diluted with the corresponding buffer to $1200 \mathrm{nM}$ and heated at $90^{\circ} \mathrm{C}$ for $5 \mathrm{~min}$, followed by slowly cooling to room temperature $(2 \mathrm{~h})$. An $8-\mu \mathrm{L}$ aliquot of the resulting sample was mixed with $2 \mu \mathrm{L}$ of $6 \times$ loading buffer and $2 \mu \mathrm{L}$ of $10 \times$ SYBR Green I. The gel electrophoresis analysis was carried out in $0.5 \times$ TBE buffer at a constant voltage at $80 \mathrm{~V}$ for $60 \mathrm{~min}$ on a gel electrophoresis instrument (BIO-RAD, USA), and the gel bands were photographed by the ChemiDoc XRS+ imaging system. 


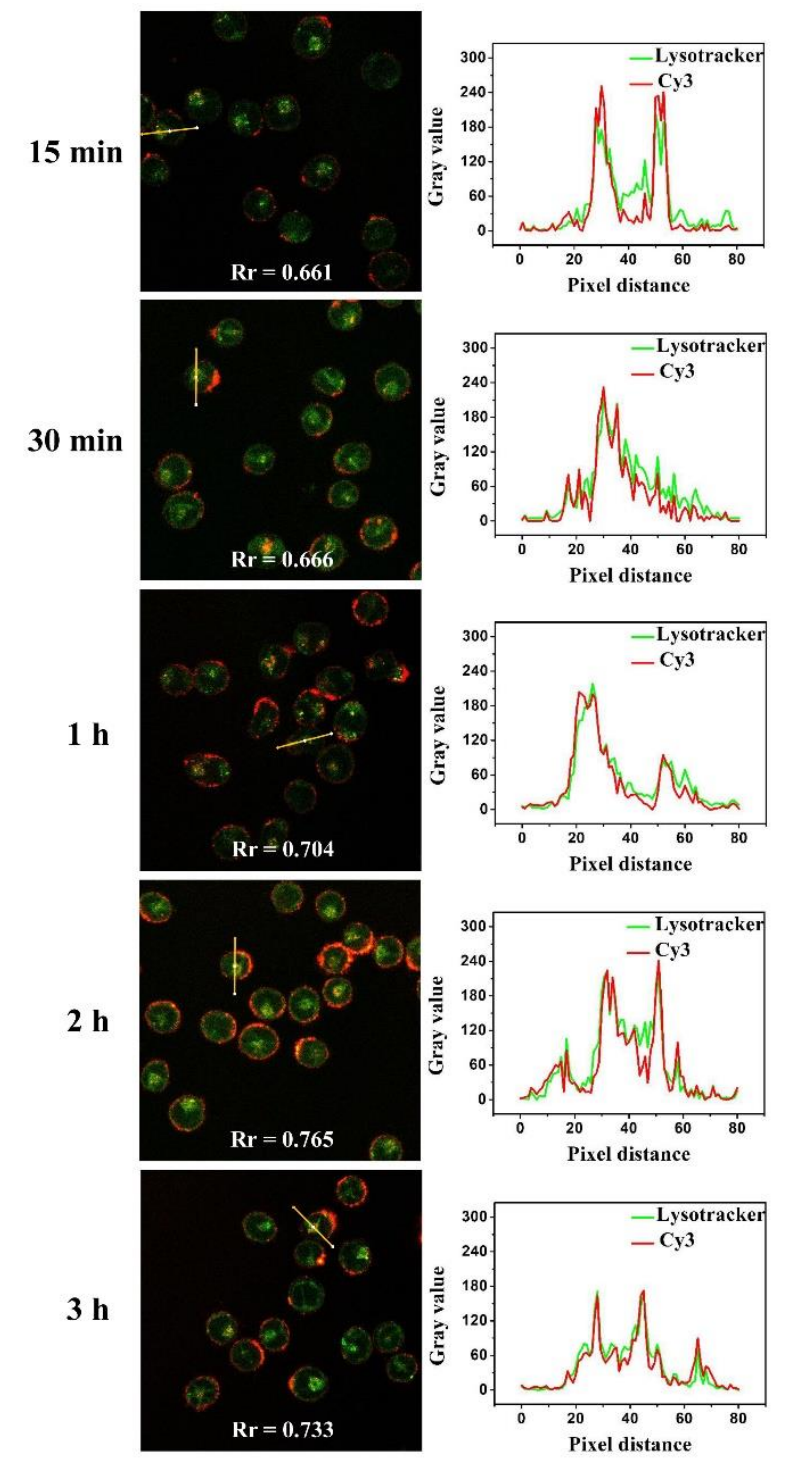

Figure S3. The confocal microscopic images (left panel) by merging Cy3 and Lysotracker channels, which are the "merged" group of Figure 2A. The colocalization analysis of corresponding Cy3 fluorescence and lysosome fluorescence performed by ImageJ was represented in the right lane. $\mathrm{Rr}$ represents the Manders overlap coefficient. Lysotraker Green (green) was used to stain the lysosomal compartment. CEM cells were incubated with Cy3-Sgc8-NSU at $37{ }^{\circ} \mathrm{C}$ for $15 \mathrm{~min}, 30$ min, $1 \mathrm{~h}, 2 \mathrm{~h}$ or $3 \mathrm{~h}$. The equivalent concentration of Cy3-labeled Sgc8 is $100 \mathrm{nM}$. 


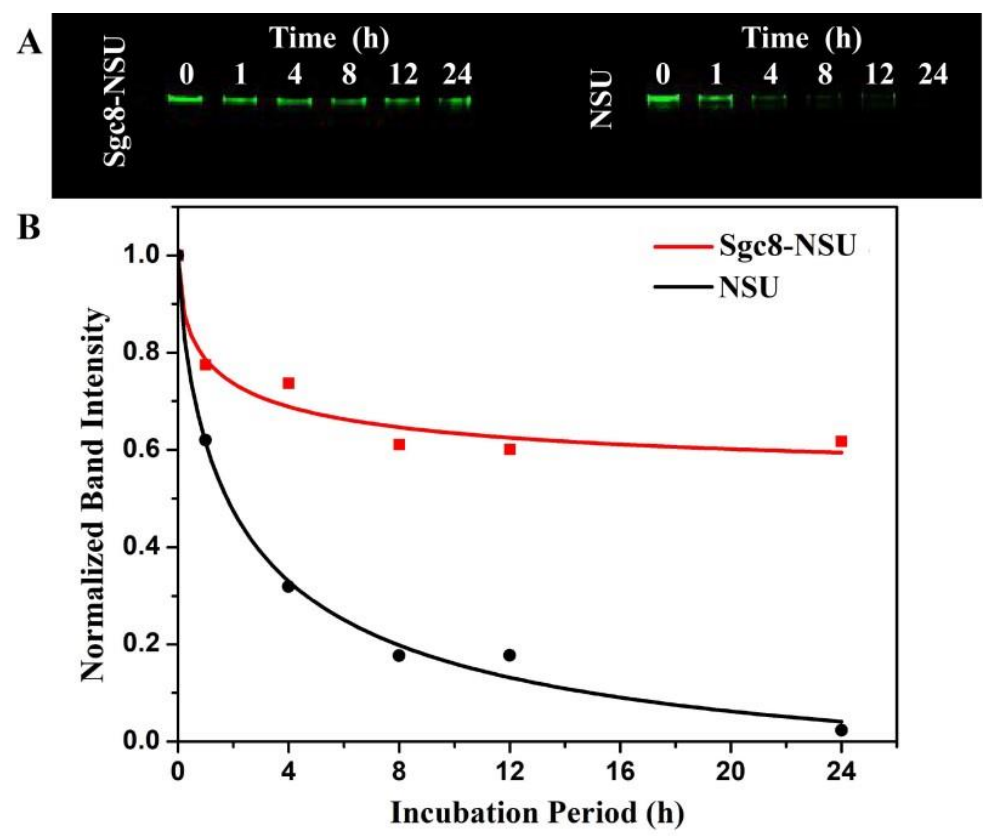

Figure S4. (A) Denaturing PAGE analysis of Sgc8-NSU and NSU incubated in FBS $(10 \% \mathrm{v} / \mathrm{v})$ at $37^{\circ} \mathrm{C}$ for $24 \mathrm{~h}$. (B) The time-dependence relative fluorescence intensity of gel electrophoresis bands.

\section{Experimental procedure:}

FAM-BPS-4 was used instead of BPS-4 for preparing Sgc8-NSU and NSU, and the assembly procedure is described in the section of 2.6. For serum stability assay, FAM-labeled Sgc8-NSU and FAM-labeled NSU (final equivalent concentration of FAM-BPS-4, $300 \mathrm{nM}$ ) were incubated with $10 \%(\mathrm{v} / \mathrm{v})$ fetal bovine serum (FBS) in RPMI medium 1640 at $37{ }^{\circ} \mathrm{C}$ for different time periods $(0$, $0.5,1,2,4,8,12$ and $24 \mathrm{~h}$ ). At the designated time point, an $10-\mu \mathrm{L}$ aliquot was taken out and mixed with $6 \mu \mathrm{L}$ of deionized formamide to terminate the degradation, followed by storing at $-20{ }^{\circ} \mathrm{C}$ until use. Finally, $10 \mu \mathrm{L}$ of the resulting solution was mixed with $10 \mu \mathrm{L}$ of $2 \times$ loading buffer and analyzed by $10 \%$ denaturing PAGE at $30 \mathrm{~mA}$ in $1 \times$ TBE buffer ( $90 \mathrm{mM}$ Tris, $90 \mathrm{mM}$ boric acid, $2 \mathrm{mM}$ EDTA, $\mathrm{pH}=7.9$ ) for $30 \mathrm{~min}$ on ice. The grayscale intensity of d-PAGE band corresponding to each time point of degradation was quantified with ImageJ. 
A

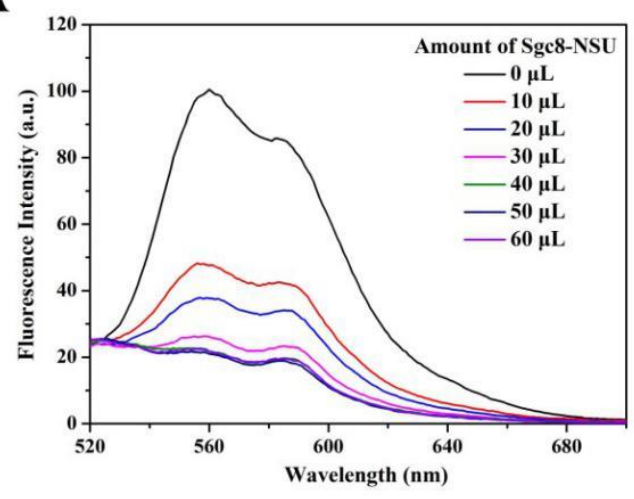

B

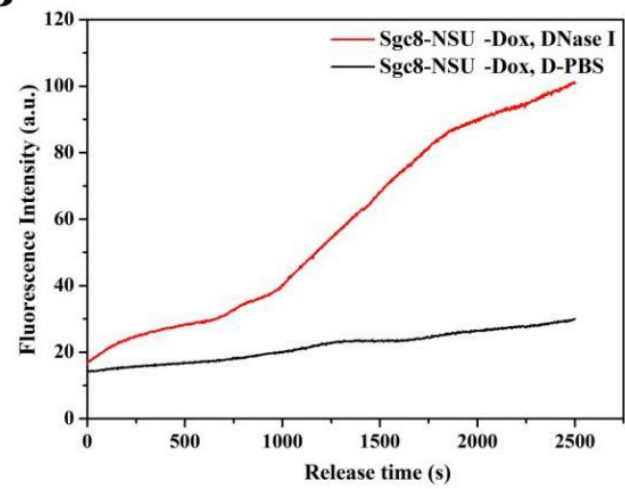

Figure S5. (A) The evaluation of drug payload capacity of Sgc8-NSU by measuring the change in the fluorescence spectrum of Dox $(0.4 \mu \mathrm{M})$ when increasing the amount of Sgc8-NSU. (B) Dox release behaviors of Sgc8-NSU-Dox in the present (red line) and absence (black line) of DNase I at room temperature.

\section{Experimental procedures:}

Different volumes of Sgc8-NSU were separately added into Dox solutions (final volume, $200 \mu \mathrm{L}$; final concentration, $0.4 \mu \mathrm{M}$ ), and the resulting solutions were incubated for $2 \mathrm{~h}$ at room temperature to load Dox into Sgc8-NSU. Then, the fluorescence spectrum of Dox was collected on F-7000 fluorescence spectrometer $(\mathrm{Ex} / \mathrm{Em}=490 / 560 \mathrm{~nm})($ Hitachi, Japan).

In order to evaluate the Dox release from Sgc8-NSU-Dox, $3 \mu \mathrm{L}$ of DNase I ( $2 \mathrm{U} / \mu \mathrm{L})$ (D-PBS used as control) was added into Sgc8-NSU-Dox $(200 \mu \mathrm{L})$, followed by immediately monitoring the fluorescence change over $2500 \mathrm{~s}$ by F-7000 fluorescence spectrometer.

\section{Discussion}

To estimate the drug loading capability of Sgc8-NSU, the number of BPS-4 incorporated in each Sgc8-NSU should be first calculated. As shown in Figure 1B, most Sgc8-NSU particles are circular or elliptical, and the AD value is more than AH. Thus, $\mathrm{Sgc} 8-\mathrm{NSU}$ is considered to be cylindrical, and the average volume is calculated as follows:

$$
\mathrm{V}=\pi \times\left(\frac{\mathrm{AD}}{2}\right)^{2} \times \mathrm{AH}=\pi \times\left(\frac{157.32}{2}\right)^{2} \times 2.78=54011.01 \mathrm{~nm}^{3}
$$

Because the self-hybridized BPS-4 probes essentially are double-stranded (ds) oligonucleotides, the volume of each probe is estimated from the following equation:

$$
\mathrm{V}_{0}=\pi \times\left(\frac{\mathrm{D}}{2}\right)^{2} \times \mathrm{s} \times \mathrm{n}=\pi \times\left(\frac{2}{2}\right)^{2} \times 0.34 \times 48=51.24 \mathrm{~nm}^{3}
$$

D-The diameter of DNA double helix is $2 \mathrm{~nm}$. ${ }^{1}$

$\mathrm{s}$-Distance between adjacent base pairs is $0.34 \mathrm{~nm} .{ }^{1}$

$\mathrm{n}$ - The base number of BPS-4 is 48 .

Because $\mathrm{V}_{0}$ is estimated based on ds oligonucleotides, the number of single-stranded BPS-4 in one Sgc8-NSU should be obtained as follows: 


$$
\frac{\mathrm{V}}{\mathrm{V}_{0}} \times 2=\frac{54011.01 \mathrm{~nm}^{3}}{51.24 \mathrm{~nm}^{3}} \times 2=2108
$$

Moreover, as shown in Figure S5A, $50 \mu \mathrm{L}$ of Sgc8-NSU, where the concentration of BPS-4 is 125 $\mathrm{nM}$, can load $0.4 \mu \mathrm{M}$ Dox (final volume of $200 \mu \mathrm{L}$ ). Thus, the drug loading capacity of Sgc8-NSU is estimated to be $1: 26982$ as shown below:

$$
\frac{\mathrm{n}_{\text {Sgc8-NSU }}}{\mathrm{n}_{\text {Dox }}}=\frac{\mathrm{V}_{\text {Sgc8 }-\mathrm{NSU}} \times \mathrm{c}_{\text {Sgc8-NSU }}}{\mathrm{V}_{\text {Dox }} \times \mathrm{c}_{\text {Dox }}}=\frac{\mathrm{V}_{\text {Sgc8-NSU }} \times \frac{\mathrm{c}_{\mathrm{BPS}-4}}{2108}}{\mathrm{~V}_{\text {Dox }} \times \mathrm{c}_{\text {Dox }}}=\frac{50 \mu \mathrm{L} \times \frac{125 \mathrm{nM}}{2108}}{200 \mu \mathrm{L} \times 0.4 \mu \mathrm{M}}=\frac{1}{26982}
$$




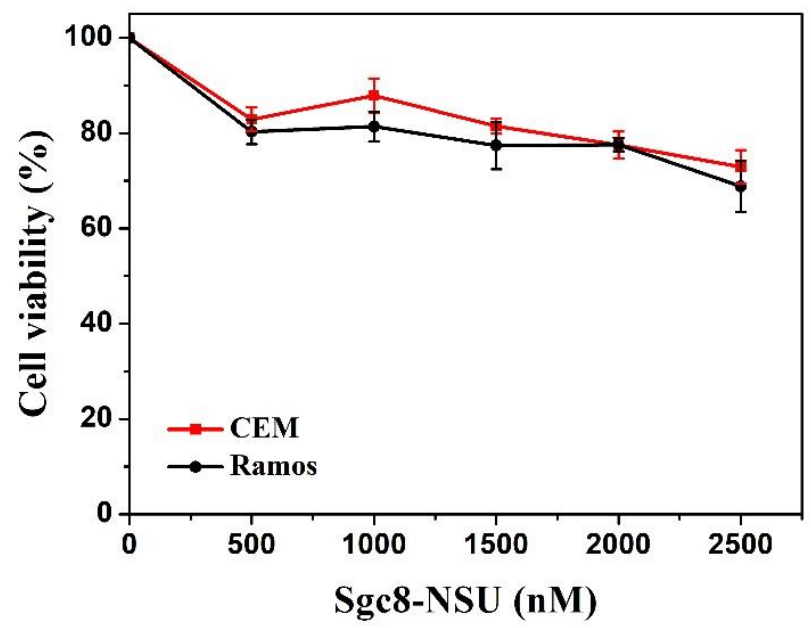

Figure S6. The biocompatibility of Sgc8-NSU evaluated by CCK-8 assay. CEM cells and Ramos cells served as target cells and control cells, respectively.

\section{Experimental procedures:}

The cytotoxicity of Sgc8-NSU to target or nontarget cells was evaluated using the Cell Counting Kit-8 (CCK-8) assay (Beyotime Bioth). After moved into 96-well plates, the cells were separately incubated with $100 \mu \mathrm{L}$ of $0.5 \times \mathrm{RPMI}$ medium 1640 containing different amounts of Sgc8-NSU for $24 \mathrm{~h}$ in a humidified atmosphere containing $5 \% \mathrm{CO}_{2}$ at $37{ }^{\circ} \mathrm{C}$. Then, the CCK-8 reagent $(10 \mu \mathrm{L})$ was added into each well and incubated for $4 \mathrm{~h}$. According to the manufacturer's instruction, the absorbance value $(450 \mathrm{~nm}$ ) was recorded using an automated plate reader (Tecan Infinite M200 PRO, Austria). 


\section{REFERENCES}

(1) Seeman, N. C., An Overview of Structural DNA Nanotechnology. Molecular Biotechnology 2007, 37 (3), 246-257. 